\title{
TIC en la escuela y rendimiento educativo: el efecto mediador del uso de las TIC en el hogar ${ }^{1}$
}

\author{
ICT at school and educational outcomes: the mediating \\ effect of the use of ICT at home
}

ISSN 1510-2432 - ISSN 1688-9304 (en línea) - DOI: https://doi.org/10.18861/cied.2018.9.1.2822

María Marta Formichella

Doctora en Economía, Universidad Nacional del Sur, Argentina. Investigadora en Economía de la Educación. Investigadora asistente, Instituto de Investigaciones Económicas y Sociales del Sur (IIESS), Consejo Nacional de Investigaciones Científicas y Técnicas (CONICET) y Universidad Nacional del Sur, Argentina. Profesora adjunta, departamento de Economía, Universidad Nacional del Sur. Ha publicado artículos en Revista CEPAL y Revista de Educación.

María Verónica Alderete

Doctora en Economía, Universidad Nacional del Sur (UNS), Argentina Investigadora en Economía de la Educación. Investigadora asistente, Instituto de Investigaciones Económicas y Sociales del Sur (IIESS). Docente, departamento de Economía, Universidad Nacional del Sur. Investigadora en Sociedad de la Información. Ha publicado artículos en Cuadernos de Economía, Information Technology for Development y Revista Desarrollo y Sociedad.

Fecha de recibido: 22/03/2017

Fecha de aceptado: 5/07/2017

\section{Resumen}

Este trabajo analiza el efecto del acceso y uso de las Tecnologías de la Información y de la Comunicación (TIC) en escuelas en el rendimiento educativo de los estudiantes de nivel medio de Uruguay. Se examina si el uso de las TIC en el hogar potencia el efecto directo del acceso y uso de las TIC en la escuela. Mediante un Modelo de Ecuaciones Estructurales (SEM) aplicado con datos de PISA 2012 para Uruguay se obtiene que el uso de y el acceso a las TIC en la escuela poseen un efecto positivo sobre el rendimiento educativo en matemática, lectura y ciencia, el cual se ve reforzado mediante el uso de las TIC en el hogar. La potencial utilidad de la investigación también reside en que las hipótesis y la metodología propuestas pueden extenderse a otros contextos similares, así como a futuras investigaciones a partir de datos de las venideras pruebas PISA.

Palabras clave: TIC, rendimiento educativo, Uruguay, SEM, PISA

\begin{abstract}
This paper analyzes the effects of the access to Information and Communication Technologies and its use at primary schools on the academic results of secondary school students in Uruguay. The authors of the present paper also assess whether the ICT use at home reinforces the ICT access and use thereof at school. By means of a Structural Equation Model and using data from PISA 2012 Uruguay, the authors' findings suggest that ICT access and use at school have a positive effect on the educational outcome in every discipline, which is strengthened by the use of ICT at home. The potential utility of the research also lies in the feasibility of the hypotheses and methodology in similar contexts. Besides, it contributes to future research to be conducted with data from forthcoming PISA tests.

Keywords: ICT, educational performance, Uruguay, SEM, PISA
\end{abstract}




\section{Introducción}

Existen diferentes motivos para afirmar que la educación es clave en los procesos de desarrollo de los individuos y de las sociedades en las que habitan (Sen, 1999) y la incorporación de las Tecnologías de la Información y la Comunicación (TIC) puede contribuir a mejorar el acceso al sistema educativo y a la calidad de los resultados escolares (McMillan Culp, Honey y Mandinach, 2003).

Es así que, en gran parte de América Latina la incorporación de TIC ha ocupado un lugar importante en la agenda educativa durante las últimas décadas. Como evidencia de ello cabe mencionar las Metas Educativas 2021 aprobadas por la Conferencia Iberoamericana de Educación (2010). Las mismas proponen la creciente utilización de computadoras en la escuela para tareas de aprendizaje como un objetivo específico dentro de la meta general de mejorar la calidad de la educación y el currículo escolar (McMillan Culp et al., 2003). En este contexto se hace necesario analizar la capacidad de uso de las TIC ya que las diferencias entre escuelas se profundizan cuando se considera el uso de los equipos y no solo el acceso a los mismos. En general se observa que el acceso a las TIC y su uso en la educación se centra en los hogares o en los laboratorios de informática más que en las aulas, lo cual impide que se optimice el efecto de la disponibilidad de las máquinas (Claro et al., 2011).

Tanto en países desarrollados como en vías de desarrollo prevalece la ambigüedad sobre los efectos de las tecnologías en el salón de clases. Algunos argumentos encontrados en la literatura para explicar esta evidencia mixta están vinculados a la consideración del nivel inicial de conocimiento de los estudiantes provenientes, en su mayoría, de la experiencia de uso en sus hogares.

Según Trucco y Espejo (2013) los docentes y estudiantes utilizan la computadora para fines o actividades relativamente básicos y destacan que el contexto socioeconómico del hogar y el capital cultural asociado son factores estructurales que determinan la capacidad de aprovechamiento de las tecnologías.

Sin embargo, que el uso de las TIC posea un impacto significativo sobre las oportunidades de los jóvenes en los ámbitos productivo, educativo, social y político depende de que dichos jóvenes desarrollen habilidades más funcionales y especializadas así como criterios para la selección y uso de la información disponible en la red. Dentro de este proceso los adultos desempeñan un papel clave en la guía y monitoreo del proceso de aprendizaje. Por esta razón constituye una preocupación el uso poco frecuente de la tecnología por parte del cuerpo docente promedio en la región latinoamericana (Trucco y Espejo, 2013).

Los datos de PISA 2003 indican que en Uruguay la diferencia de acceso a una computadora en el hogar entre estudiantes del cuartil superior de ingreso y el cuartil inferior es mucho mayor que la diferencia de acceso a un computador en el colegio. En particular, la brecha es de 70 puntos porcentuales en el hogar y de 13 puntos porcentuales en el colegio (Claro, 2010). Esto pone en evidencia la relevancia del uso de las TIC en el hogar.

La magnitud de las brechas anteriormente mencionadas ha disminuido como consecuencia de las políticas públicas (Claro, 2010), en especial de la implementación del Plan Ceibal. El mismo consistió en otorgar una computadora portátil y acceso a Internet de forma gratuita a cada alumno (modelo 1 a 1). Al cabo de tres años (2007-2010) logró que todos los estudiantes de primaria y secundaria accedieran al mismo (Vaillant, 2013) y que el porcentaje de hogares con acceso a computadoras del Uruguay aumentara 
significativamente. Sin embargo, esto no eliminó la brecha existente entre el porcentaje de hogares con computadora y el porcentaje de hogares con computadora y conexión a Internet (Dodel, 2010; Rivoir y Lamschtein, 2012).

Pero esto no ha sucedido únicamente en Uruguay. Si bien solo un tercio de los países de la región ha diseñado una política formal de TIC en educación, la mayoría ha desarrollado iniciativas a través de programas y proyectos (Sunkel y Trucco, 2010).

En este contexto el presente trabajo tiene como objetivo analizar la importancia del acceso y uso de las TIC como factores determinantes del rendimiento educativo. La hipótesis general que se pretende examinar es la siguiente: el acceso y uso de las TIC en la escuela produce un efecto positivo en el rendimiento educativo de los estudiantes de nivel medio y dicho efecto es potenciado por el uso de las TIC en el hogar. En este sentido la hipótesis propone que el uso de las TIC en el hogar es una variable mediadora entre el acceso y uso de las TIC en la escuela y el rendimiento educativo. Con el fin de testearla se utiliza un Modelo de Ecuaciones Estructurales (SEM) con datos de PISA 2012 correspondientes a Uruguay. El trabajo se estructura de la siguiente manera. En la sección 2 se presenta el marco teórico y los antecedentes empíricos sobre el tema. En la siguiente se exponen la metodología, datos y variables utilizados. En la sección 4 se presentan los resultados hallados y en la última se exponen las conclusiones.

\section{Marco teórico y antecedentes sobre el tema}

Para realizar un análisis adecuado del rol de las TIC en educación es necesario distinguir entre acceso y uso de las mismas. El acceso a las TIC se refiere a la disponibilidad de las nuevas tecnologías y dispositivos tales como si el estudiante (o el establecimiento educativo) posee computadoras, Internet y telefonía celular, entre otros. El uso de las TIC implica las capacidades de utilización que están vinculadas a la manera y los motivos por los que las personas utilizan las computadoras e Internet (Van Deursen y Van Dijk, 2009). Así, lo que verdaderamente importa no es únicamente el acceso a las TIC sino que exista la capacidad de utilizarlas efectivamente. Actualmente se evidencian grandes diferencias en el grado de acceso, las formas de uso y los procesos de adopción de las tecnologías entre las distintas jurisdicciones y escuelas.

En este sentido, el aprovechamiento de las TIC por parte de un estudiante no solo depende de las oportunidades disponibles sino también del tipo de actividad realizada mediante las nuevas tecnologías. Esto define dos tipos de brecha digital: la primera, la diferencia en el acceso y la segunda, las diferencias en el uso de las TIC y la capacidad de beneficiarse de ellas (Hargittai, 2002; Robinson, DiMaggio y Hargittai, 2003).

Con el fin de comprender la relación entre uso de TIC y aprendizaje es preciso analizar los tipos de uso de estas tecnologías y la relación de estos con conceptos y destrezas de disciplinas específicas. Los resultados hallados en el área afirman que el aprendizaje con TIC en el aula solo es factible y puede resultar exitoso si existen condiciones escolares y pedagógicas específicas tales como un acceso adecuado a recursos TIC, docentes que integran las TIC a la currícula y a la experiencia escolar y condiciones institucionales favorables (Claro, 2010). 
Así, con el advenimiento de las TIC surgió una serie de conceptos para comprender los diferentes niveles que las personas alcanzan en cuanto al acceso y uso de las TIC. En torno al uso se destaca el concepto de alfabetización digital (Sánchez, Olivares y Alvarado, 2013). En un primer momento el concepto hizo referencia al nivel de aprendizaje y al nivel de habilidades necesarias para hacer uso de las TIC o al conjunto mínimo de competencias necesarias para operar eficazmente con herramientas tecnológicas como la computadora (Buckingham, 2006; Gilster, 1997). Posteriormente, no solo incluyó las competencias básicas para hacer uso de la tecnología sino también el uso adecuado de las TIC en términos de desarrollo de otras habilidades. Eshet-Alkalai (2004) considera la alfabetización digital como algo más que la mera capacidad de operar con un software o dispositivo digital, ya que incluye una serie de complejos cognitivos, motores, sociológicos y habilidades emocionales que los usuarios necesitan para funcionar eficazmente en entornos digitales.

El proceso de enseñanza-aprendizaje no se desarrolla exclusivamente en la escuela sino también en otros espacios como el hogar o el entorno socio-comunitario. De esta manera surge la consideración de los procesos pedagógicos que se gestan en el interior del hogar como un factor clave para explicar el nivel de habilidades desarrolladas y alcanzadas por los estudiantes.

El acceso a las TIC en el hogar desempeña un papel clave para el desarrollo de las habilidades digitales que actualmente son requeridas en la escuela (Kuhlemeier y Hemker, 2007). Con el objeto de apoyar a los alumnos a dar sus "primeros pasos" en el mundo virtual interesan tanto las posibilidades de acceso a las TIC y al entorno digital en el hogar como la pertenencia a un entorno familiar con el know-how o el conocimiento de este nuevo lenguaje digital (Buckingham, 2006).

Con datos provenientes del cuestionario de familiaridad computacional para TIC (Information and Communication Technology Familiarity Questionnaire) para PISA 2009 respecto a los usos se comprueba que los estudiantes utilizan las nuevas tecnologías más frecuentemente en el hogar que en la escuela lo cual es compatible con otros estudios a nivel internacional (Claro et al., 2011). De este modo, en el contexto de la alfabetización digital, si bien las TIC amplían las posibilidades pedagógicas y didácticas, la escuela pierde protagonismo en el proceso formativo de los individuos ya que los alumnos traen del hogar los códigos para desarrollar este nuevo mundo tecnológico, en ocasiones con más conocimiento que los propios docentes (Kuhlemeier y Hemker, 2007; Umar y Jalil, 2012; Fundación Telefónica, 2012; Claro et al., 2012).

Más allá de la relevancia de las TIC en el hogar expresada en los párrafos anteriores, varios estudios han intentado demostrar la existencia de una correlación positiva entre la infraestructura TIC disponible en los hogares y las escuelas y el desempeño de los estudiantes. Sin embargo, aún no hay consenso al respecto (Balanskat, Blamire y Kefala, 2006).

Por una parte existe un grupo de investigaciones que encuentra un impacto positivo y significativo de las variables TIC sobre algunos resultados educativos de nivel medio. Por otra parte hay un grupo de trabajos que no encuentra efecto alguno de las variables TIC $y$, en menor medida, algunos localizan una incidencia negativa de las mismas sobre los logros académicos. 
Mediante un modelo de ecuaciones estructurales Aristizábal et al. (2009) estudian el efecto de las TIC en el hogar y en la escuela sobre el rendimiento educativo. Con este fin emplean datos de las pruebas PISA 2006 y 2009 en Colombia y encuentran que las TIC escolares alcanzan el mayor impacto. De igual modo, Spiezia (2010) estudia el efecto de las TIC sobre el rendimiento educativo de nivel medio con datos de PISA 2006 para el total de los países participantes y destaca el rol del uso de las TIC en el hogar por sobre su uso en las escuelas. Como resultado cuestiona las políticas de incorporación de computadoras como herramienta de aprendizaje en la escuela.

Por su parte, mediante el método de Propensity Score Matching (PSM) y datos de PISA 2012 Alderete y Formichella (2016) estudian el impacto del Programa Conectar Igualdad en Argentina que entrega una computadora portátil a los alumnos de educación media de gestión pública. Esto implica que el Programa permite el acceso a las TIC tanto en las escuelas como en los hogares. Encuentran un efecto positivo del mismo sobre los logros escolares aunque sostienen la necesidad de explotar las potencialidades del Programa, en especial en lo que se refiere al uso de las TIC ya que los resultados no trascienden a los del mero acceso.

Por el contrario, Angrist y Lavy (2002) estudian el caso de Israel y encuentran que el uso de herramientas informáticas en los procesos de enseñanza-aprendizaje escolares no tiene efectos significativos. Asimismo, Goolsbee y Guryan (2006) examinan el efecto del Programa E-Rate consistente en un subsidio para el uso de Internet en las escuelas de California (Estados Unidos) y no evidencian cambios en el rendimiento educativo.

Por su parte, Sprietsma (2012) estima el efecto del acceso y uso de PC e Internet sobre los resultados educativos de matemática y lectura en estudiantes de octavo grado en Brasil y halla un impacto negativo de las TIC sobre el rendimiento escolar. Barrera-Osorio y Linden (2009) estudian el efecto de las TIC sobre el rendimiento escolar en Colombia. Encuentran que la disponibilidad de computadoras obtenidas del Programa "Computadoras para la Educación" no tiene efecto significativo sobre los resultados de aprendizaje como consecuencia del uso inadecuado de las mismas. Asimismo, Muñoz y Ortega (2014) analizan el impacto de dos planes de incorporación de las TIC en la enseñanza implementados en Chile y encuentran que los programas no alcanzaron resultados significativos sobre los logros educativos.

Algunas investigaciones han abordado el impacto de las TIC -consideradas exclusivamente en el hogar- sobre los resultados educativos. Formichella et al. (2015) hallan diferencias estadísticamente significativas en el rendimiento educativo promedio derivadas del uso de las TIC en el hogar con datos de Argentina. Asimismo, con datos de países de América Latina Botello y Rincón (2014) encuentran que el acceso a una computadora y/o Internet en el hogar puede incrementar el puntaje promedio de los estudiantes. En la misma línea, Notten y Kraaykamp (2009) hallan una correlación positiva y significativa entre la disponibilidad de TIC en el hogar y los puntajes obtenidos en los exámenes de PISA 2006 en ciencia para 53 países.

Por el contrario, algunos estudios no encuentran un efecto significativo de las TIC en el hogar sobre los resultados educativos (Malamud y Pop-Eleches, 2010 en Romania; Wittwer y Senkbeil, 2008 en Alemania). 
Finalmente, en cuanto al caso particular de Uruguay, Tansini y Aguilar (2011) identifican los efectos que la disponibilidad de computadoras en el hogar genera sobre el desempeño escolar de estudiantes de escuelas públicas de nivel primario de Montevideo (capital de Uruguay). Los autores confirman que junto con el acceso a computadoras en el hogar existen otras variables relacionadas con el mismo tales como el entorno cultural y educativo familiar que son relevantes para el éxito escolar. Por su parte, Rodríguez Zidán y Téliz (2011) explican que la implementación del Plan Ceibal para educación secundaria se corresponde con un nuevo modelo de gestión y de propuesta metodológicas.

De Melo et al. (2013) estudian el efecto del Plan Ceibal y no encuentran un impacto significativo de éste sobre los resultados en las áreas de matemática y lectura debido a un uso inadecuado de las computadoras pues las mismas se utilizan principalmente para buscar información por Internet. Asimismo, De Melo et al. (2013a) también concluyen que el Plan Ceibal no tuvo efecto sobre el rendimiento de las pruebas de matemática y lectura, ya fuera empleando los datos a nivel agregado o por nivel socioeconómico. Tampoco encuentran un efecto sobre otro tipo de competencias no cognitivas.

Trucco y Espejo (2013) elaboran un modelo para identificar los factores asociados al grado de experticia TIC de estudiantes de nivel primario de Uruguay mediante un modelo multinivel. Los autores hallan que el uso en la escuela aumenta la probabilidad de ser un usuario experto en comparación con el uso en el hogar. Sin embargo resaltan la relevancia de que la madre sea usuaria de Internet y la importancia de desarrollar estrategias para la promoción del uso de las computadoras en las familias con proyectos que promuevan el trabajo conjunto de escuelas, familias y servicios de Ceibal. Por último, si bien Martínez, Leite y Monteiro (2016) reconocen la contribución de las TIC al desarrollo económico y social, lo que justifica su presencia en la educación escolar, destacan la necesidad de cambios en la formación inicial de los maestros.

En suma, en este apartado se han destacado antecedentes acerca del rol de las TIC en relación a la educación, tanto en el hogar como en la escuela pues existe evidencia, con sus matices, acerca de la importancia del acceso y uso de las mismas en ambos entornos.

\section{Datos, metodología y variables}

\section{Datos}

Con el fin de llevar a cabo el trabajo empírico se utilizan datos del Programme for International Student Assessment (PISA) realizado por la OCDE (OECD por su sigla en inglés) desde el año 2000 y que viene aplicándose cada tres años con el fin de evaluar los resultados educativos de estudiantes de diferentes países. En este caso se utiliza información correspondiente a Uruguay en el año 2012.

OCDE evalúa la medida en que los alumnos de 15 años de edad, que se hallan próximos a concluir su educación obligatoria, poseen las competencias necesarias para desarrollar su vida adulta. Por ello, la prueba es implementada en chicos de esa edad que se encuentren escolarizados (OCDE, 2014).

Cabe señalar que no se obtiene un único valor de respuesta para cada alumno en cada una de las competencias evaluadas -matemáticas, ciencias y lengua- sino que los resultados de cada estudiante se presentan por medio de cinco valores plausibles, los cuales representan su conjunto de capacidades (OCDE, 2009). 
Dado que el objetivo de PISA no es evaluar a cada alumno sino a la población estudiantil en general cada estudiante no resuelve todos los ítems de la prueba sino algunos. Luego se estima cómo hubiera respondido en la totalidad de los mismos y de allí surgen los cinco valores plausibles correspondientes a cada individuo en cada una de las competencias analizadas (OCDE, 2009).

Cabe señalar que el equipo de PISA indica que la información debe ser analizada globalmente, por lo que no es correcto calcular promedios por alumno por competencia. El manual de PISA de la OCDE explica que la forma adecuada de estimar cualquier estadístico es llevar a cabo la metodología de estimación para cada uno de los cinco valores individualmente y luego calcular el promedio del estadístico en cuestión (OCDE, 2009).

En este trabajo se siguen los pasos indicados por OCDE (2009). Por ello se plantea un modelo y se replica para cada valor posible estimándose en forma aislada cada una de las competencias estudiadas en PISA. Luego, a partir de los resultados obtenidos se calculan los parámetros como el promedio de dichos resultados.

\section{Metodología y variables}

Este trabajo analiza la relación causal entre el acceso y uso de las TIC en la escuela, el uso de las TIC en el hogar y el rendimiento educativo. Dentro de este marco la hipótesis propuesta es que el uso de las TIC en el hogar ejerce un rol mediador. Es decir, la pregunta de investigación plantea si el acceso y uso de las TIC en la escuela poseen un impacto directo sobre el rendimiento educativo e indirecto en el hogar, mediado por el uso de las TIC. Asimismo, se plantea que existe influencia de otras variables sobre el rendimiento educativo, las que actúan como control dentro del modelo (más adelante se describe cada una de ellas).

Con el objeto de contrastar empíricamente tales relaciones propuestas se emplea el Análisis de Ecuaciones Estructurales (SEM). Los modelos estructurales se definen como aquellos en los cuales los parámetros son tanto de naturaleza descriptiva como causal (Bollen, 1989). Por ello, en estos modelos un elemento fundamental es la presencia de relaciones causales entre las variables que los componen.

En este sentido, los modelos de ecuaciones estructurales constituyen una familia de modelos estadísticos multivariantes a través de los cuales se estiman el efecto y las relaciones entre múltiples variables. Estos modelos se destacan por: la representación gráfica de las relaciones causales, el planteo de hipótesis sobre los efectos causales entre las variables y la concatenación de efectos entre variables.

A diferencia de los modelos de regresión tradicionales, los modelos de ecuación estructurales poseen la ventaja de disponer de una mayor flexibilidad y permitir incluir errores de medida tanto en las variables criterio (dependientes) como en las variables predictoras (independientes), siendo por esto menos restrictivos que aquellos (Ruiz, Pardo y San Martín, 2010). De esta manera permiten establecer tanto el tipo de relaciones entre las diversas variables como su dirección, para luego estimar los parámetros especificados en las relaciones propuestas a nivel teórico. Por tal razón se denominan frecuentemente modelos confirmatorios ya que el principal interés es confirmar las relaciones propuestas sobre la base de la teoría mediante el análisis de una muestra particular (Ruiz et al., 2010). 
Por un lado, las tres condiciones que deben satisfacer los modelos de ecuaciones estructurales para definir una relación causal son: aislamiento, asociación y dirección de la causalidad. Ninguna de estas condiciones es fácil de obtener, en particular la condición de aislamiento que exige que la relación causal (causa-efecto) no esté afectada por otros factores. Por otro lado, el establecimiento de una relación causal entre las variables debe estar apoyada por el marco teórico del modelo y no por su estimación con datos de corte transversal (Ruiz et al., 2010).

En los modelos SEM suelen intervenir dos tipos de variables: observadas y latentes. Por naturaleza, las variables latentes no son susceptibles de medición ni de una definición precisa. Por el contrario, las variables observadas representan las características observables de un fenómeno y se pueden medir de forma directa. Por esta razón, las variables latentes se construyen a partir de combinaciones lineales de las variables observadas.

En general, los modelos de ecuaciones estructurales se describen mediante un diagrama de trayectorias y un sistema de ecuaciones. En el diagrama de trayectorias las variables observadas se grafican mediante rectángulos y las latentes se representan mediante elipses o círculos. Asimismo, el sistema de ecuaciones está conformado por dos secciones importantes: el modelo de medida y el modelo estructural. Para un correcto desarrollo de la técnica deben evaluarse tanto el modelo de medida como el modelo estructural (Barclay, Higgins y Thompson, 1995).

Por un lado, mediante el modelo de medida se definen los indicadores o variables observables que construyen la variable o constructo latente así como los errores de medición de cada una de dichas variables. Por otro lado se estima el modelo de relaciones estructurales que se compone de los efectos y relaciones entre los constructos, los cuales serán normalmente variables latentes.

La expresión general de los modelos de ecuaciones estructurales se presenta mediante las siguientes ecuaciones matriciales (Jöreskog, 1973):

$$
\eta=B \eta+\xi+\varsigma
$$

donde $\mathrm{n}$ indica el vector de variables aleatorias latentes endógenas de dimensión m $\times 1 ; \xi$ describe el vector de variables aleatorias latentes exógenas de dimensión $\mathrm{n} \times 1$; $B$ constituye la matriz de coeficientes que determinan las relaciones entre las variables endógenas y tiene una dimensión $\mathrm{m} \times \mathrm{m}$; $\tau$ es la matriz de coeficientes de dimensión $\mathrm{m} \times \mathrm{n}$ determinando las relaciones entre las variables exógenas y cada una de las endógenas, es decir, indica los efectos de $\xi$ sobre $n, y \_$representa al vector de perturbaciones o errores. Junto con el modelo estructural se presenta el modelo de medida que se construye a partir de dos ecuaciones. Por un lado, una mide las relaciones entre las variables latentes endógenas y sus variables observadas:

$y=\Lambda_{y} \eta+\varepsilon$

donde y es el vector de $p$ variables observables $(p \times 1)$; $\wedge y$ representa la matriz de coeficientes e indica las relaciones entre las variables latentes y las observadas ( $\mathrm{x} \times \mathrm{m}$ ), también conocida como matriz de cargas y $\boldsymbol{\varepsilon}$ es el vector de errores (px1). 
Por otro lado, la segunda ecuación del modelo de medida rige las relaciones entre las variables latentes exógenas y sus variables observables:

$x=\Lambda_{x} \eta+\delta$

tal que $x$ es el vector de $p$ variables observables ( $q \times 1)$; $\wedge x$ es la matriz de coeficientes 0 de cargas que representan las relaciones entre las variables latentes y las observadas (q $\mathrm{x} \mathrm{m})$ y $\delta$ es el vector de errores (qx1).

Mediante el método de Máxima Verosimilitud se estima el modelo, el que supone el cumplimiento del supuesto de normalidad. Sin embargo, una ventaja de los modelos SEM es que son robustos al incumplimiento de este supuesto (Schermelleh-Engel, Moosbrugger y Müller, 2003). Para su estimación se ha utilizado el paquete estadístico Stata 12.

El marco conceptual se representa en la siguiente figura:

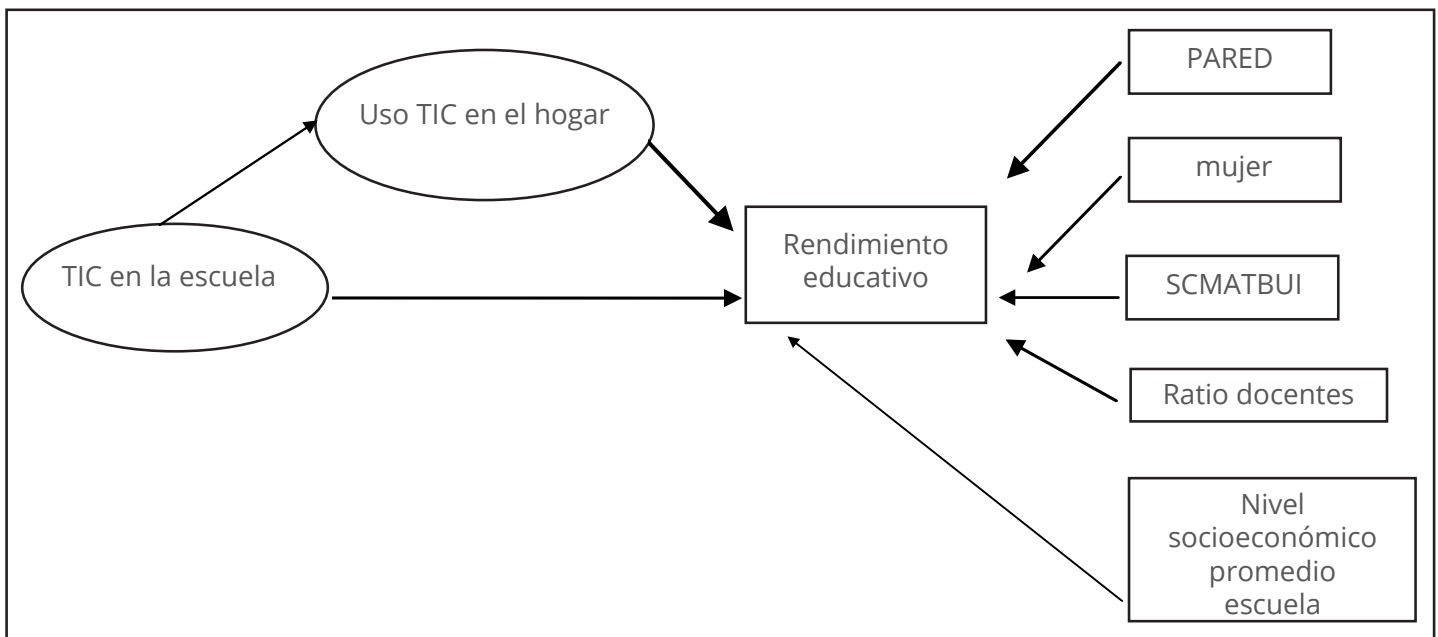

Figura 1. Marco conceptual.

Fuente: Elaboración propia.

Asimismo, en función de la pregunta de investigación planteada en este trabajo se define el modelo estructural. Este comprende dos partes, siendo dos las variables endógenas del mismo: el Uso de las TIC en el hogar y el Rendimiento Educativo. A continuación, la Tabla 1 indica las variables que son incluidas en el modelo estructural a estimar.

Tabla 1. Orden explicativo de las variables propuestas.

\begin{tabular}{|l|l|}
\hline Variables independientes (exógenas) & Variables dependientes (endógenas) \\
\hline Latentes: & $\begin{array}{l}\text { Observadas: } \\
\text { TIC_escuela }\end{array}$ \\
Observadas: & Latentes: \\
PARED & Uso_ hogar \\
MUJER & \\
SCMATBUI & \\
Ratio de docentes por alumno & \\
Nivel socioeconómico promedio escolar & \\
\hline
\end{tabular}

Fuente: elaboración propia 


\section{Donde:}

- TIC_escuela es una variable latente que representa el acceso a y uso de las TIC en el ámbito escolar (más adelante se detalla cómo está conformada).

- Uso_hogar es una variable latente que representa la frecuencia y modo de utilización de las tecnologías en el hogar (más adelante se detalla cómo está conformada)

- PARED es una variable continua, un índice construido por el equipo de PISA a partir de los años de educación de los padres tomando el mayor entre padre y madre. Representa precisamente el nivel educativo de los progenitores: cuanto mayor es el valor del índice mayor educación han recibido.

- MUJER es una variable dicotómica que toma valor uno si el estudiante es de género femenino y cero en caso contrario.

- SCMATBUI es una variable continua, un índice construido por el equipo de PISA a partir de diferentes indicadores escolares. Representa la calidad de la infraestructura del centro escolar.

- Ratio de docentes por alumno es una variable continua que representa la cantidad de docentes certificados y de dedicación completa por alumno en un determinado colegio secundario.

- Nivel socioeconómico promedio escolar (NSP) es una variable continua que surge como promedio del índice ESCS² de cada estudiante en una determinada escuela. ESCS es un índice construido por el equipo de PISA que representa el status social y económico del hogar de los alumnos. A mayores valores, mayor nivel socioeconómico.

- Rendimiento educativo es una variable continua que representa el puntaje obtenido por cada alumno en las pruebas PISA en las competencias de ciencias, matemática y lectura (PVSCIE es el valor plausible en ciencias, PVMATH en matemáticas y PVREAD en lengua). El modelo estructural plantea las siguientes hipótesis:

$\mathrm{H} 1$ : el acceso y uso de las TIC en la escuela ejerce un efecto significativo y positivo sobre el uso de las TIC en el hogar.

$\mathrm{H} 2$ : el uso de las TIC en el hogar ejerce un efecto significativo y positivo sobre el rendimiento educativo.

H3: el acceso y uso de las TIC en la escuela ejerce un efecto significativo y positivo en el rendimiento educativo.

H4: el nivel educativo de los padres del estudiante ejerce un efecto significativo y positivo en el rendimiento educativo.

H5: las mujeres poseen mejor rendimiento en lengua, mientras que los varones en ciencias y matemáticas.

H6: el nivel socioeconómico promedio de la escuela a la que asiste el individuo tiene un efecto significativo y positivo en el rendimiento educativo.

H7: la calidad de la infraestructura de la escuela a la que asiste el alumno tiene un efecto significativo y positivo en el rendimiento educativo.

H8: la cantidad de docentes certificados y de trabajo de dedicación completa por alumno ejerce un efecto significativo y positivo sobre el rendimiento educativo.

El modelo estructural es usualmente descripto mediante un diagrama de trayectoria o camino. De este modo, la Figura 1 distingue los determinantes del rendimiento educativo y las relaciones entre rendimiento educativo, acceso y uso de las TIC en la escuela, y uso de las TIC en el hogar.

Como puede observarse en dicha figura el modelo estimado está conformado por una variable latente endógena mediadora referida al Uso de las TIC en el hogar (denominada Uso_hogar) y una variable latente exógena (acceso y uso de TIC en la escuela). Estas variables son libres del efecto de los errores de medición, tal como ocurre con el modelo de factor común del análisis factorial. Por lo tanto, en primer lugar se estima un modelo de 
medida para crear cada constructo latente (denominado TIC_escuela) a partir de variables observables (exógenas) que permitan representar y evaluar el acceso y uso de las TIC, es decir, variables que reflejan disponibilidad o actividades y tipos de uso de las mismas. Las variables observables que componen el constructo TIC_escuela son:

- PC_escuela. Cantidad de computadoras disponibles en la escuela con fines educativos. - Internet_escuela. Cantidad de computadoras disponibles en la escuela que están conectadas a Internet.

- USESCH. Índice elaborado por el equipo de PISA que representa el Uso de las TIC en la escuela. Se construye a partir de 7 ítems o indicadores sobre actividades realizadas con las computadoras en la escuela. De cada indicador existe una escala de respuesta de 5 categorías que varía desde Nunca o casi nunca a Todos los días.

Las que componen el constructo Uso_hogar son:

- Uso tarea Web. Frecuencia con que el estudiante utiliza la red fuera de la escuela para estudiar o realizar tareas.

- Uso tarea PC. Frecuencia con que el estudiante utiliza el ordenador fuera de la escuela para estudiar o hacer tareas.

\section{Resultados}

En la presente sección se presentan los resultados hallados a partir del Modelo de Ecuaciones Estructurales propuesto.

En primer lugar se analiza la construcción de las variables latentes Uso de TIC en el hogary TIC en la escuela. Las mismas fueron elaboradas con el fin de testear la hipótesis propuesta: “El uso de TIC en el hogar potencia el efecto que produce la introducción de TIC en el proceso de enseñanza-aprendizaje en el interior de las escuelas". Es decir, la variable Uso de TIC en el hogar se ha propuesto como variable mediadora (ver Figura 2).
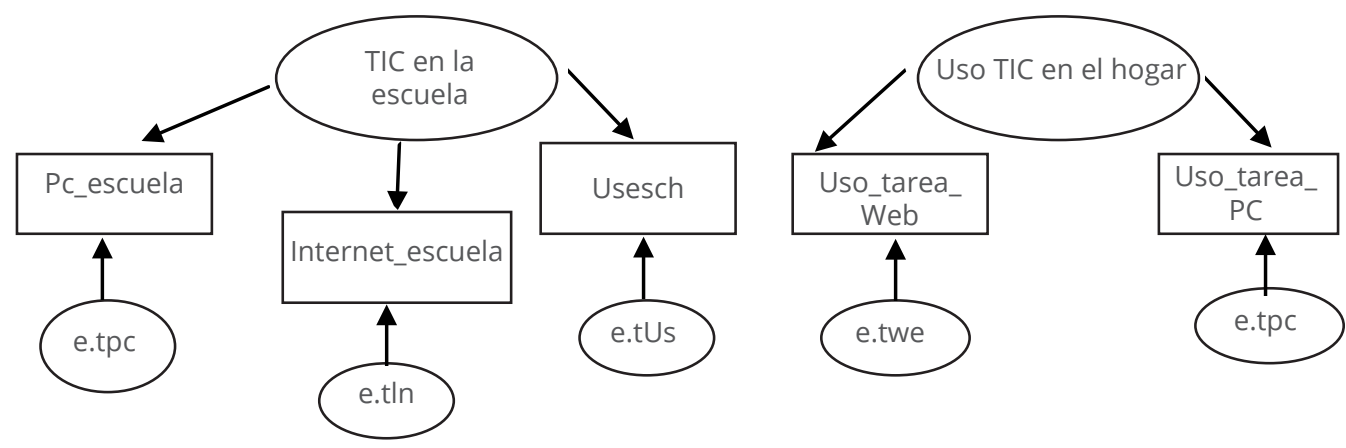

Donde e.t son los errores de cada una de las variables observadas.

Figura 2. Modelo de medida de Uso TIC en la escuela y fuera de la escuela.

Fuente: Elaboración propia

El modelo posee una parte denominada Modelo de medida que muestra precisamente en qué magnitud los ítems que conforman cada constructo son fiables. En la Tabla 2 pueden observarse los factores que construyen las variables latentes Uso TIC en el hogar y TIC en escuela. En la mencionada tabla se muestran los resultados para el modelo teniendo en cuenta cada una de las competencias estudiadas por el Programa PISA y puede concluirse 
que el conjunto de variables observadas dan significado al constructo que fue diseñado a partir de la teoría. Esto es así debido a que los coeficientes que acompañan a dichas variables han resultado ser todos estadísticamente diferentes de cero.

Tabla 2. Modelo de Medida de las variables Uso TIC en el hogar y TIC en escuela.

\begin{tabular}{|l|l|l|l|l|}
\hline \multicolumn{2}{|l|}{ Constructos latentes } & Modelo con \\
Variables & $\begin{array}{l}\text { Factores que las } \\
\text { componen }\end{array}$ & $\begin{array}{l}\text { Modelo con } \\
\text { ciencias }\end{array}$ & $\begin{array}{l}\text { Modelo con } \\
\text { lectura }\end{array}$ \\
\hline \multirow{4}{*}{$\begin{array}{l}\text { TIC en la } \\
\text { escuela }\end{array}$} & PC_escuela & 1 (restringido) & 1 (restringido) & 1 (restringido) \\
\cline { 2 - 5 } & Internet_escuela & $0.85504796^{* * *}$ & $0.83369525^{* * *}$ & $0.84433008^{* * *}$ \\
\cline { 2 - 5 } & Usesch & $0.42200494^{* * *}$ & $0.44053385^{* * *}$ & $0.42470482^{* * *}$ \\
\hline \multirow{3}{*}{$\begin{array}{l}\text { Uso TIC en el } \\
\text { hogar }\end{array}$} & Uso_tarea_Web & 1 (restringido) & 1 (restringido) & 1 (restringido) \\
\cline { 2 - 5 } & $\begin{array}{l}\text { Uso_tarea_ } \\
\text { PC }\end{array}$ & $0.87216524 * * *$ & $0.858768525 * * *$ & $0.8305913^{* * *}$ \\
\hline
\end{tabular}

Nota: ***Significatividad al 1\%;**Significatividad al 5\%; *Significatividad al 10\%.

Fuente: Elaboración propia a partir de la base de datos PISA 2012 (OCDE).

El segundo fragmento del modelo propuesto se denomina Modelo Estructural, el cual a su vez se divide en dos partes, una correspondiente a la ecuación estructural Uso TIC en el hogar y la segunda referida a la ecuación estructural Rendimiento educativo. La primera parte (A) está representada en la Tabla 3. Como puede observarse, los efectos de la variable latente TIC en la escuela sobre el constructo Uso TIC en el hogar son positivos y estadísticamente significativos al estimar, separadamente, el modelo con información del rendimiento educativo en matemática, ciencias y lengua. Es decir, el acceso y uso de las TIC en la escuela aumentan el uso de las TIC en el hogar. Por ende puede señalarse que, en base los datos aquí analizados, se confirma la hipótesis número 1 expuesta en el trabajo $(\mathrm{H} 1)$.

Tabla 3. Modelo estructural. Parte A.

\begin{tabular}{|l|l|l|l|}
\hline \multirow{2}{*}{$\begin{array}{l}\text { Variable } \\
\text { explicativa }\end{array}$} & \multicolumn{4}{|l|}{ Variable explicada: Uso_TIC en el hogar } \\
\cline { 2 - 4 } & $\begin{array}{l}\text { Modelo con } \\
\text { rendimiento en } \\
\text { matemática }\end{array}$ & $\begin{array}{l}\text { Modelo con } \\
\text { rendimiento en } \\
\text { ciencias }\end{array}$ & $\begin{array}{l}\text { Modelo con } \\
\text { rendimiento en } \\
\text { lengua }\end{array}$ \\
\cline { 2 - 4 } & Coeficiente & Coeficiente & Coeficiente \\
\hline TIC en la escuela & $0.75919724 * * *$ & $0.7497609 * \star *$ & $0.75627108^{\star * *}$ \\
\hline
\end{tabular}

Nota: ***Significatividad al 1\%;**Significatividad al 5\%; *Significatividad al 10\%.

Fuente: Elaboración propia a partir de la base de datos PISA 2012 (OCDE).

La segunda parte del modelo estructural (B) puede observarse en la Tabla 4. En principio cabe señalar que las variables TIC en la escuela y Uso en el hogar muestran una incidencia positiva y estadísticamente significativa sobre el rendimiento en las tres competencias analizadas. Esto confirma las hipótesis número 2 y $3(\mathrm{H} 2$ y H3) aquí propuestas.

El resultado obtenido indica que el uso de las TIC en el hogar potencia el efecto que ejercen el acceso y uso de las TIC en la escuela sobre los logros educativos cognitivos. En síntesis, la variable Uso en el hogar desempeña un papel mediador entre la variable TIC en la escuela y las variables de rendimiento escolar. 
Asimismo, y a partir de la comparación de los valores de los coeficientes que acompañan a las variables recientemente mencionadas puede afirmarse que el rol de las TIC en la escuela tiene un efecto directo más relevante sobre el rendimiento en matemática, no observándose diferencias entre lengua y ciencias respecto a su influencia sobre los logros educativos. En relación al efecto indirecto, el cual refleja en qué medida el rol de las TIC en la escuela se ve potenciado por el uso de las mismas en el hogar, no presenta diferencias en cuanto a disciplinas cuando se observa la implicancia de las TIC en la escuela sobre el uso de estas en el hogar (Tabla 3). Sin embargo, sí se evidencian discrepancias en cuanto a la incidencia del uso de las TIC en el hogar sobre el rendimiento (Tabla 4). En este caso, el efecto mediador del uso de las TIC en el hogar es más importante en el caso de lengua, siguiéndole en relevancia ciencias y quedando en último lugar matemática. El resultado de este efecto es diferente al descripto en los efectos directos.

Tabla 4. Modelo estructural. Parte B.

\begin{tabular}{|c|c|c|c|}
\hline \multirow{3}{*}{ Variables explicativas } & \multicolumn{3}{|c|}{ Variables explicadas } \\
\hline & Matemática & Lengua & Ciencias \\
\hline & Coeficiente & Coeficiente & Coeficiente \\
\hline TIC_escuela & $35.348648 * * *$ & $32.798754 * \star \star$ & $32.75316 * * *$ \\
\hline Uso_Hogar & $3.6297552 * * *$ & $6.697403 * \star *$ & $6.0096552 * * *$ \\
\hline PARED & $1.8519276 * \star \star$ & $1.3968954 * \star \star$ & $2.018396 * * *$ \\
\hline MUJER & $-17.327656 * \star \star$ & $28.042522 * \star \star$ & $-5.550622 * \star$ \\
\hline SCMATBUI & $6.648286 * \star *$ & $6.6656648 * * *$ & $4.9873568 * * *$ \\
\hline RATIO_DOCENTES & 166.8626 ** & $-199.67878 * *$ & -84.239038 \\
\hline NSP & $48.795218 * * *$ & $51.090748 * \star *$ & $50.597846 * * *$ \\
\hline
\end{tabular}

Nota: ***Significatividad al 1\%;**Significatividad al 5\%; *Significatividad al 10\%.

Fuente: Elaboración propia a partir de la base de datos PISA 2012 (OCDE).

Asimismo cabe señalar el efecto de las variables incluidas en el modelo como control. Como se muestra en la tabla anterior, cuanto mayor es el nivel educativo de los padres mayor es el rendimiento en las tres competencias analizadas. Asimismo, el género posee un rol importante observándose un mejor rendimiento de las mujeres en lengua y un rendimiento superior de los varones en ciencias y matemáticas. Así se confirman las hipótesis 4 y 5 (H4 y H5) anteriormente planteadas.

Con respecto a las variables que caracterizan a las escuelas, hay dos que han resultado tener coeficientes estadísticamente significativos en las tres competencias estudiadas: SCMATBUI y Nivel Socioeconómico Promedio del alumnado. Cuanto mejor es la infraestructura de la escuela (lo cual se correlaciona positivamente con los recursos físicos y educativos de las misma) y cuanto mayor es el status socioeconómico de los compañeros de escuela, mayor es el rendimiento del individuo. Esto confirma las hipótesis 6 y 7 (H6 y H7).

Es así que la hipótesis acerca del rol del número de docentes calificados y con dedicación completa en relación a la cantidad de alumnos (hipótesis $8, \mathrm{H} 8$ ) solo se ha verificado en el caso de matemática. La variable ratio_docentes no resultó ser estadísticamente significativa para el caso de ciencias y su resultado es contrario al esperado para el caso de lengua (un análisis pormenorizado de esto excede el alcance de los objetivos del presente trabajo). 
Por último cabe presentar los resultados correspondientes a la Bondad de Ajuste del Modelo. En los modelos SEM la misma se evalúa por medio de varios indicadores, un test de significancia y varios estadísticos descriptivos. En la tabla 5 pueden observarse diferentes medidas referidas al ajuste del modelo presentado.

Tabla 5. Bondad de ajuste del modelo.

\begin{tabular}{|l|l|l|l|}
\hline \multirow{2}{*}{ Estadístico } & \multicolumn{3}{|l|}{ Valor } \\
\cline { 2 - 4 } & Matemática & Lengua & Ciencias \\
\hline chi2_ms(35) & 718.194 & 728.3106 & 675.9426 \\
\hline p > chi2 & 0 & 0 & 0 \\
\hline RMSEA & 0.08 & 0.08 & 0.07 \\
\hline SRMR & 0.05 & 0.05 & 0.05 \\
\hline
\end{tabular}

Fuente: elaboración propia.

En principio cabe señalar que valores de chi2 altos no representan una adecuada bondad de ajuste del modelo (se espera que dichos valores sean cercanos a cero). No obstante, cuando se trata de muestras grandes -como sucede en el caso aquí presentado- el valor de este indicador tiende a ser elevado $y$, por ello, no se recomienda tenerlo en cuenta para evaluar el modelo (Schermelleh-Engel, Moosbrugger y Müller, 2003). Por tal motivo se propone observar el error de aproximación cuadrático medio (RMSEA) y la raíz cuadrada media residual (RMR) de Jöreskog y Sörbom (1989). El primero indica que si su valor es menor a 0,10 el límite inferior del intervalo de confianza del 90\% es inferior a 0,05, y por lo tanto el ajuste es aceptable. Como puede observarse, el RMSEA es menor a 0,10 en las tres competencias estudiadas por lo que, de acuerdo a este indicador, puede decirse que la bondad de ajuste del modelo es aceptable.

Respecto a la raíz cuadrada media residual (RMR) cabe señalar que es una medida general de "Maldad" de Ajuste (en contraposición a bondad de ajuste) que se basa en los residuos ajustados. La dificultad que plantea este indicador es que es dependiente de la escala de las variables. Por ello, para salvar esta cuestión se introduce la raíz cuadrada media residual estandarizada (SRMR). En este caso, un valor de cero indica un ajuste perfecto y por ello se acepta como regla que existe un buen ajuste del modelo si el valor de la SRMR es inferior a 0.05 (Bentler, 1995). Como se evidencia en la Tabla 5, el indicador SRMR no supera dicho valor en ninguna de las competencias estudiadas. Por ende, se afirma que la bondad de ajuste del modelo es buena.

\section{Conclusiones}

En el presente artículo se ha analizado el rol de las Tecnologías de la Información y de la Comunicación (TIC) en relación al logro de resultados educativos. A partir de datos de la prueba PISA 2012 para el caso de Uruguay se ha intentado hacer un aporte en dicho sentido. Una de las principales contribuciones de este trabajo a la literatura consiste en analizar la relación causal entre el acceso y uso de las TIC en la escuela y el rendimiento educativo destacando el rol del hogar como mediador. Existen escasos trabajos en Uruguay que, desde una metodología cuantitativa, examinen la relación entre TIC y educación. 
Para ello se ha estimado un Modelo de Ecuaciones Estructurales (SEM) con el fin de estudiar la incidencia del acceso y uso de las TIC en las escuelas sobre los resultados educativos en matemáticas, lengua y ciencias y se ha hecho hincapié en responder a la pregunta de si el uso de las TIC en el hogar potencia el efecto de la utilización de TIC en las escuelas. Por un lado, a partir de la muestra de datos utilizada y la metodología empleada se ha encontrado evidencia a favor de la existencia de un efecto directo del acceso y uso de las TIC en las escuelas sobre el rendimiento educativo. Por otro lado, también se ha verificado la hipótesis de que la variable Uso de TIC en el hogar posee un rol mediador al potenciar dicho efecto.

Estos resultados no concuerdan con la evidencia hallada en el contexto del Plan Ceibal, lo que puede deberse a diferentes razones. Por un lado, los datos de PISA se vinculan mayormente al nivel medio mientras que los datos utilizados por De Melo et al. (2013, 2013a) corresponden a nivel primario y secundario. Por otro lado, dicha investigación utiliza datos de panel, es decir que incorporan el factor tiempo, lo cual aquí no es posible. Finalmente cabe aclarar que este trabajo no se concentra en el Plan Ceibal sino que examina el efecto de las TIC más allá del mismo pues considera, por ejemplo, el impacto del uso de Internet en el hogar. A pesar de que el Plan Ceibal ha mejorado el acceso a Internet en las escuelas y zonas aledañas, la brecha existente en el acceso a Internet en el hogar (Dodel, 2010; Rivoir y Lamschtein, 2012) podría explicar la divergencia en los resultados hallados por otros autores.

Asimismo, desde el punto de vista de las políticas implementadas en Uruguay los resultados hallados corroboran la importancia de los programas públicos como el Plan Ceibal. Dado que el uso de las TIC en el hogar ejerce un rol mediador, los programas de difusión de las TIC en las escuelas deben incluir como medida no solo el traslado de las computadoras al hogar sino también garantizar la conectividad en todos los hogares.

En trabajos futuros se intentará dar mayor robustez a estas conclusiones repitiendo el análisis para otros países que hayan sido evaluados por las pruebas PISA y en los cuales también se haya utilizado el cuestionario complementario acerca de acceso y uso de las Tecnologías de la Información y la comunicación para PISA 2012 (Information and Communication Technology Familiarity Questionnaire).

\section{Referencias biliográficas}

Alderete, M.V. y Formichella, M.M. (2016). Análisis del efecto de las TIC en el rendimiento educativo: el caso del Programa Conectar Igualdad en Argentina. Revista de la CEPAL, 119, 89-107.

Angrist J. y Lavy, V. (2002). New evidence on classroom computers and pupil learning. The Economic Journal, 112: 735-765.

Aristizábal, G., Caicedo, M. y Escandón, D. (2009). Las Tecnologías de la Información y Comunicación como determinante en el rendimiento académico escolar, Colombia 20062009. Disponible en: http://2012.economicsofeducation.com/user/pdfsesiones/011.pdf

Balanskat, A., Blamire, R. y Kefala, S. (2006). The ICT Impact Report: a review of studies of ICT impact on schools in Europe. Bruselas, Comunidades Europeas. Disponible en: http://unpan1.un.org/intradoc/groups/public/documents/unpan/unpan037334.pdf 
Barclay, D., Higgins, R. y Thompson, R. (1995). The partial least squares (PLS) approach to causal modelling: Personal computer adoption and use as an illustration. Technology Studies, Special Issue on Research Methodology 2(2), 285-309.

Barrera-Osorio, F. y Linden, L. (2009). The Use and Misuse of Computers in Education. Evidence from a Randomized Experiment in Colombia. Policy Research Working Paper 4836. Disponible en: http://documents.worldbank.org/curated/en/346301468022433230/pdf/WPS4836.pdf

Bentler, P.M. (1995). EQS Structural Equations Program Manual. Encino, C.A: Multivariate Software, Inc.

Botello, A.L.H. y Rincón, G.A. (2014). La influencia de las TIC en el desempeño académico de los estudiantes en América Latina: Evidencia de la prueba PISA 2012. Memorias Virtual Educa, Memorias VE2014: Lima, Perú, 2014. Disponible en: http://recursos.portaleducoas. org/sites/default/files/VE14.146.pdf

Buckingham, D. (2006). Defining digital literacy - What do young people need to know about digital media? Medienbildung In Neuen Kulturräumen, 59-71.

Claro, M. (2010). Impacto de las TIC en los aprendizajes de los estudiantes. Estado del arte. Documento de proyecto CEPAL, Santiago de Chile.

Claro, M., Espejo, A., Jara, I. y Trucco, D. (2011). Aporte del sistema educativo a la reducción de las brechas digitales. Una mirada desde las mediciones PISA. Documento de proyecto CEPAL, Santiago de Chile.

Claro, M., Preiss, D., San Martín, E., Jara, I., Hinostroza, E., Valenzuela, S. \& Nussbaum, M. (2012). Assessment of 21 st century ICT skills in Chile: Test design and results from high school level students. Computers \& Education, 1042-1053.

De Melo, G., Machado, A., Miranda, A. y Viera, M. (2013). Profundizando en los efectos del Plan Ceibal. Serie Documentos de Trabajo, DT 12/2013. Instituto de Economía, Facultad de Ciencias Económicas y Administración, Universidad de la República, Uruguay. Disponible en: http://fcea.edu.uy/Jornadas Academicas/2013/file/MESAS/Economia\%20de\%20la\%20 educacion_plan\%20ceibal/Profundizando\%20en\%20los\%20efectos\%20del\%20Plan\%20 Ceibal.pdf

De Melo, G., Machado, A., Miranda, A. y Viera, M. (2013). Impacto del Plan Ceibal en el aprendizaje. Evidencia de la mayor experiencia OLPC. Serie Documentos de Trabajo, DT 13/2013. Instituto de Economía. Facultad de Ciencias Económicas y Administración, Universidad de la República, Uruguay.

Dodel, M. (2010). Acceso a las TIC en hogares del Uruguay urbano: logros y desafíos para la (sic) asentar los pisos de una integración digital. Presentación en las IX Jornadas de Investigación de la Facultad de Ciencias Sociales, Universidad de la República, Montevideo, 2010.

Eshet-Alkalai, Y. (2004). Digital Literacy: A Conceptual Framework for Survival Skills in the Digital Era. Journal of Educational Multimedia and Hypermedia, 13(1), 93-106. 
Formichella, M.M, Alderete, M.V.y Di Meglio, G.A. (2015). El acceso a las TIC en el hogar como determinante del rendimiento educativo en el nivel medio: un análisis para Argentina. En López, M.R. y Graves, J. (coord.). Investigaciones de Economía de la Educación N¹0, Capítulo 3, 357-375. Madrid, Asociación de Economía de la Educación.

Fundación Telefónica (2012). Alfabetización digital y competencias informacionales. Ariel, Madrid.

Gilster, P. (1997). Digital Literacy. New York: Wiley Computer Pub.

Goolsbee, A. y Guryan, J. (2006). The impact of Internet subsidies in public schools. The Review of Economics and Statistics, 88(2), 336-347.

Hargittai, E. (2002). Second-level digital divide: difference in people's online skills. First Monday 7 (4). Disponible en: http://firstmonday.org/htbin/cgiwrap/bin/ojs/index.php/fm/ article/view/942/864.

Jöreskog, K. G. (1973). A general method for estimating a linear structural equation system. In A. S. Goldberger and O. D. Duncan (Eds.), Structural Equation Models in the Social Sciences (pp. 85-112). New York: Academic Press.

Jöreskog, K. G. y Sörbom. D. (1989). SPSS LISREL VII and PRELIS User's Guide and Reference, 1st ed. Chicago: SPSS, Inc.

Kuhlemeier, H. y Hemker, B. (2007). The impact of computer use at home on students' Internet skills. Computers \& Education 49, 460-480.

Malamud, O. y Pop-Eleches, C. (2010). Home computer use and the development of human capital. NBER Working Paper 15814. Disponible en: www.nber.org/papers/w15814.

Martínez, R., Leite, C. y Monteiro, A. (2016). TIC y formación inicial de maestros: oportunidades y problemas desde la perspectiva de estudiantes. Cuadernos de Investigación educativa, 7(1), 69-92.

McMillan Culp, K., Honey, M. y Mandinach, E. (2003). A Retrospective on Twenty Years of Education Technology Policy. Washington, D.C., U.S. Department of Education, Office of Educational Technology.

Muñoz, R. y Ortega, J. (2014). ¿Tienen la banda ancha y las TICs un impacto positivo sobre el rendimiento escolar? Evidencia para Chile. El trimestre económico, 82(325), 53-87.

Notten, N. y Kraaykamp, G. (2009). Home media and science performance: a cross-national study. Educational Research and Evaluation 15, 367-384.

OECD (2009). PISA Data Analysis Manual. SPSS: SAS, Second Edition. París: OECD Publishing. OECD (2014). PISA Technical Report. París: OECD Publishing.

Rivoir, A. L. y Lamschtein, S. (2012). Cinco años del Plan Ceibal. Algo más que una computadora para cada niño. Montevideo: Unicef, Uruguay. 
Robinson, J.P., DiMaggio, P. y Hargittai, E. (2003). New Social Survey Perspectives on the Digital Divide. IT \& Society 1 (5), 1-22.

Rodríguez Zidán, E. y Téliz, F.A. (2011). La larga marcha hacia la revolución digital en las escuelas. Análisis de la implementación de políticas TIC en educación a partir de la experiencia del Plan Ceibal y el modelo uno a uno en Uruguay. Perspectivas en Políticas Públicas, 4(7), 27-60.

Ruiz, M.A., Pardo, A. y San Martín, R. (2010). Modelos de ecuaciones estructurales. Papeles del Psicólogo 31 (1), 34-45.

Sánchez, J., Olivares, R. y Alvarado, P. (2013). Variables asociadas al hogar y resultados de la prueba SIMCE TIC. En Catalina Araya Lima (cood). Desarrollo de habilidades digitales para el siglo XXI en Chile: ¿Qué dice el SIMCE TIC?, 55-79.

Schermelleh-Engel, K., Moosbrugger, H. y Müller, H. (2003). Evaluating the fit of structural equation models: tests of significance and descriptive goodness-of-fit measures. Methods of Psychological Research Online, 8, 2, 23-74.

Sen, A. (2000). Desarrollo y libertad. Ed. Planeta.

Spiezia, V. (2010). Does computer use increase educational achievements? Student-level Evidence from PISA. OECD Journal: Economic Studies. Disponible en: http://www1.oecd.org/ eco/labour/49849896.pdf

Sprietsma, M. (2012). Computers as pedagogical tools in Brazil: a pseudo-panel analysis. Education Economics, 20(1), 19-32.

Sunkel, G. y Trucco, D. (2010). Nuevas tecnologías de la información y la comunicación para la educación en América Latina: riesgos y oportunidades. CEPAL. Serie políticas sociales $\mathrm{N}^{0} 167$.

Tansini, R. y Aguilar, R. (2011). Computers at home and schoolchildren's outcome in Uruguay. Trabajo presentado en Latin American and Caribbean Economic Association (LACEA) \& Latin American Meeting of the Econometric Society (LAMES). Disponible en: http://www. webmeets.com/lacealames/2011/prog/viewpaper.asp?pid=18

Trucco, D. y Espejo, A. (2013). Principales determinantes de la integración de las TIC en el uso educativo. El caso del Plan Ceibal del Uruguay. CEPAL. Serie políticas sociales Nº177.

Umar, I.N. y Jalil, N.A. (2012). ICT skills, practices and barriers of its use among secondary school students. Procedia - Social and Behavioral Sciences, 46, 5672-5676.

Vaillant, D. (2013). Las políticas TIC en los sistemas educativos de América Latina. Caso Uruguay. Programa TIC y Educación Básica. Fondo de las Naciones Unidas para la Infancia (Unicef), Argentina.

Van Deursen, A., y Van Dijk, J. (2009). Using the Internet: Skill related problems in users' online behavior. Interacting with Computers, 21(5-6), 393-402. Disponible en: https://www. utwente. $\mathrm{nl} / \mathrm{nl} / \mathrm{bms} / \mathrm{cw} /$ bestanden/Using\%20the $\% 20$ Internet-\%20Skill\%20related\%20 problems.pdf 
Wittwer, J. y Senkbeil, M. (2008). Is students' computer use at home related to their mathematical performance at school? Computers \& Education 50, 1558-1571.

\section{(Endnotes)}

${ }^{1}$ Los autores agradecen al proyecto "Identificación de políticas educativas eficaces en la mejora de la calidad educativa" y al proyecto "Innovación Social Sostenible: innovaciones y políticas públicas en perspectiva regional".

1Para mayores detalles sobre la construcción de los índices PARED, SCMATBUI y ESCS ver PISA 2012 Technical Report (OECD, 2014). 